\title{
Germinación y crecimiento de Vicia magellanica: Implicancias para la restauración de áreas incendiadas en el norte de la Patagonia andina
}

\author{
Melisa Blackhall ${ }^{1, \otimes}$; Ana Villán ${ }^{2} \&$ Miriam E. Gobbi ${ }^{2}$ \\ ${ }^{1}$ Laboratorio Ecotono, INIBIOMA, CONICET-Universidad Nacional del Comahue. Bariloche, Argentina. ${ }^{2}$ Departamento de \\ Biología, Universidad Nacional del Comahue. Bariloche, Argentina.
}

\begin{abstract}
Resumen. Conocer la dinámica de regeneración de las especies pioneras luego de incendios es primordial para diseñar herramientas de protección y restauración de ambientes degradados por fuego. El estudio de la autoecología de las especies nativas puede aportar información muy útil para aplicar en planes de manejo y en medidas de restauración pasiva o activa. En este trabajo se presentan resultados obtenidos a partir de ensayos de germinación y crecimiento de Vicia magellanica (Fabaceae), en suelo quemado, con y sin agregado de compost, a fin de a) identificar factores asociados a los incendios que se puedan relacionar con los patrones de regeneración post-fuego de esta especie, y además b) discutir el potencial de esta especie para su aplicación en restauración de áreas incendiadas del NO de la Patagonia. En general, los porcentajes de germinación de $V$. magellanica superaron el $90 \%$ de germinación total promedio en la mayoría de los tratamientos (control, aplicación de cenizas, escarificación física, estratificación fría). Sin embargo, la aplicación de calor seco, asociado a altas temperaturas edáficas durante un incendio $\left(100{ }^{\circ} \mathrm{C}\right.$ y $\left.120^{\circ} \mathrm{C}\right)$ tuvo un efecto negativo sobre la germinación. Los resultados del ensayo de crecimiento en el que se evaluó el desempeño de $V$. magellanica en un posible escenario de restauración activa luego de un incendio mostraron que las condiciones edáficas asociadas a un suelo quemado permitieron un desarrollo satisfactorio de los individuos. En suelos quemados sin adición de compost se observó una mayor biomasa aérea y subterránea, y un mayor número de flores, en comparación con las plantas con agregado de compost. Esto supondría una ventaja para colonizar áreas con suelos degradados por fuego. Vicia magellanica presenta numerosas características que favorecerían su uso en estrategias de revegetación post-fuego en bosques y matorrales del noroeste patagónico aplicando siembra directa a campo, y particularmente en los primeros estadios luego del incendio.
\end{abstract}

[Palabras clave: regeneración, semillas, temperatura, escarificación, cenizas, compost]

\begin{abstract}
Aвstract. Germination and growth of Vicia magellanica: Potential applications on post-fire restoration in north Andean Patagonia. Knowing the regeneration dynamics of pioneer species after fires is essential to design tools for the protection and restoration of environments degraded by fire. Autoecology studies of native species can contribute with valuable information for planning land management and passive and/or active restoration actions. We conducted essays of seed germination and plant growth (utilizing burned soils with and without incorporation of compost) with the purpose of a) identifying factors associated to fire that could be related to post-fire regeneration patterns of Vicia magellanica (Fabaceae), and, as well, b) discussing the potential role of this species in the active restoration of burned areas of northwest Patagonia. In general, percentages of germination exceeded $90 \%$ of total germination for almost all treatments (control, application of ashes, physical scarification, cold stratification). However, the exposition of seeds to dry heat, associated to the elevated soil temperatures during fire occurrence $\left(100{ }^{\circ} \mathrm{C}\right.$ and $\left.120^{\circ} \mathrm{C}\right)$, showed a negative effect on seed germination. The results of the plant growth experiment evaluating the performance of V. magellanica in a possible active restoration scenario after a fire showed that the edaphic conditions associated with a burnt soil allowed a satisfactory development of individuals. Plants growing in burned soils without biosolids compost presented a higher aboveground and belowground biomass and a higher number of flowers, in comparison to plants growing in burned soils without compost. These characteristics might confer V. magellanica an advantage for colonizing burned degraded soils. Vicia magellanica presents several properties that could support the use of this species for post-fire revegetation strategies in forest and shrublands of northwest Patagonia, principally by applying direct sowing in the field, and particularly during early post-fire stages.
\end{abstract}

[Keywords: regeneration, seeds, temperature, scarification, ash, biosolids compost]

Editora asociada: Vanessa Lencinas
Recibido: 7 de Mayo de 2020

Aceptado: 23 de Septiembre de 2020 


\section{INTRODUCCIÓN}

En el noroeste patagónico, y a lo largo del gradiente ambiental que abarca desde la estepa árida a los bosques andinos húmedos y lluviosos, los incendios, tanto de origen natural como antrópicos, constituyen uno de los disturbios más influyentes sobre la estructura y la composición de las comunidades vegetales (Veblen et al. 2011). En la región, el fuego actúa a diferentes escalas y puede modificar las condiciones a nivel de micrositio (Blackhall et al. 2015) y también moldear los paisajes actuales (Kitzberger et al. 2016). En el presente escenario de cambio climático global se pronostica un aumento en la ocurrencia de incendios para la región de los bosques templados del noroeste patagónico durante la segunda mitad del siglo XXI. Esto constituye una amenaza para los ecosistemas naturales y las zonas de interfase urbanoforestal (Mariani et al. 2018).

Las principales consecuencias directas del fuego en los bosques y los matorrales son la disminución de la cobertura vegetal de todos los estratos y la degradación del suelo, que conllevan, por ejemplo, a cambios a nivel microclimático o en la biodiversidad de la flora y la fauna (Bond and van Wilgen 1996; Blackhall et al. 2015). En los bosques templados del noroeste patagónico, en donde los suelos son mayormente de origen volcánico, durante los primeros años luego de transcurrido un incendio es posible registrar una reducción de la materia orgánica de las capas superficiales del suelo, un aumento de la repelencia al agua, del $\mathrm{pH}$ y de la conductividad eléctrica, y variaciones fuertes en la disponibilidad de nutrientes (Alauzis et al. 2004; Morales et al. 2013). En particular en bosques quemados de Nothofagus pumilio en el noroeste de la Patagonia se ha observado que si bien los niveles iniciales de algunos nutrientes (e.g., fósforo) pueden aumentar con el aporte de los materiales del incendio y la liberación de recursos, luego estos niveles disminuyen, ya que el suelo queda sin capacidad de retención de humedad y nutrientes (Alauzis et al. 2004; Varela 2005). Sumado a esto, la falta de cobertura vegetal induce a un gran aumento de la erosión debida a las precipitaciones y la escorrentía. El fuego afecta también el banco de semillas, sobre todo en las capas más superficiales, donde las semillas se ven sometidas a altas temperaturas y su capacidad de germinar se ve alterada (Fenner and Thompson 2005), lo que afecta la recuperación de la vegetación. La germinación y el establecimiento de las especies vegetales en suelos recientemente quemados se puede ver afectada de forma positiva o negativa por la exposición de las semillas a las altas temperaturas, por el aumento del $\mathrm{pH}$ por acumulación de cenizas, por el efecto físicoquímico del humo sobre las cubiertas externas e internas de las semillas, por la posible escarificación física por las partículas del suelo o por el aumento de la irradiación solar debido a la falta de cobertura vegetal (Keeley and Fotheringham 2000). Al quemarse la materia orgánica durante un incendio, el suelo presenta una mayor proporción de material inorgánico que, sumado a la mayor exposición al viento (i.e., por una disminución de la cobertura vegetal), puede inducir a una mayor escarificación física de las semillas expuestas. Además, a nivel de micrositio, la drástica disminución de la cobertura vegetal puede exponer a las semillas a una mayor amplitud térmica (Blackhall et al. 2015) y, por lo tanto, existe una mayor exposición a temperaturas ambientales extremas tanto cálidas como frías.

En este contexto, conocer la dinámica de regeneración de las especies pioneras luego de incendios resulta relevante para diseñar herramientas de protección y restauración de ambientes degradados. En particular, el estudio de la autoecología de especies nativas puede aportar información muy útil para aplicar en planes de manejo y en medidas de restauración pasiva o activa. Los lineamientos básicos relacionados con proyectos de revegetación resaltan la importancia del uso de semillas de especies nativas, en especial de procedencia local o regional (Mortlock 2000). Los beneficios de usar especies nativas locales para revegetar y restaurar son variados; estos beneficios incluyen la adaptación a las condiciones ambientales locales, el aporte a la reconstrucción de las redes de interacción con otras especies vegetales y animales nativas (i.e., polinización, facilitación, mutualismos) y la amplia y económica accesibilidad a las fuentes de semillas, plántulas o renovales en áreas aledañas y en viveros locales (Mortlock 2000; Prach et al. 2007).

Por otro lado, se determinó que es factible acelerar de forma significativa la restauración de la estructura compleja del suelo mediante el uso de especies leguminosas (Fabaceae), en comparación con la aplicación directa y continua de nitrógeno inorgánico (del Moral et al. 2007). Estas especies clave son muy usadas para aumentar la disponibilidad de 
nitrógeno en suelo y recuperar las condiciones edáficas de sitios disturbados (del Moral et al. 2007; Wang et al. 2010). En bosques y matorrales del noroeste patagónico existen varias especies de leguminosas nativas, pero en particular Vicia nigricans y $V$. magellanica fueron registradas en abundancia durante la regeneración temprana en sitios post-fuego (Gobbi et al. 1995; Varela et al. 2006; Blackhall et al. 2008; Raffaele et al. 2011; Morales et al. 2013). Existe poca información documentada sobre la autoecología de estas especies nativas en general, y en menor medida en cuanto a la presencia de sus semillas en los bancos de semillas. Entre estos escasos registros, Varela et al. (2006) registró la presencia de V. nigricans en bancos de semillas transitorios en mantillos de bosques de N. pumilio post-fuego de seis años de edad. En el caso de $V$. magellanica, la documentación publicada acerca de las semillas de la especie es incluso menor o nula.

Vicia magellanica se caracteriza por ser una especie herbácea, anual o bianual (Correa 1984). Muestra un gran desarrollo del sistema radicular con raíces finas muy ramificadas que, como la mayoría de las especies de la familia Fabaceae, presenta numerosos nódulos con leghemoglobina (observación personal), asociada con la capacidad de fijar nitrógeno atmosférico (Downie 2005), y a su vez, indirectamente, al incremento de nitrógeno disponible para otras especies vegetales (van der Maarel 2005). Todas estas características hacen de $V$. magellanica una de las especies con gran potencial para ser aplicada en la rehabilitación de áreas degradadas; por ejemplo, en los primeros meses tras un incendio podría prevenir la excesiva degradación del suelo. Otras especies del género Vicia son usadas con éxito como plantas enriquecedoras y protectoras del suelo en la preparación de terrenos para cultivar (Baigorria et al 2013) o como especies facilitadoras en el establecimiento y crecimiento de especies arbóreas nativas en la restauración de áreas degradadas (Gómez Ruiz 2011).

Con el fin de identificar si existen factores asociados a los incendios que puedan relacionarse con los patrones de regeneración post-fuego de $V$. magellanica, especie sobre la cual no existe información registrada, y discutir el potencial de esta especie para su aplicación en restauración de áreas incendiadas de la Patagonia, en este trabajo se plantearon como objetivos a) evaluar la germinación de $V$. magellanica, b) evaluar el efecto directo e indirecto del fuego sobre la respuesta germinativa de sus semillas que pudieran modificar su germinación, y c) evaluar la supervivencia y desarrollo de las plántulas en sustrato con aporte de compost de biosólidos en suelos afectados por fuego reciente. A partir de estos resultados se espera aportar nuevos datos para describir la autoecología de $V$. magellanica.

\section{Materiales y Métodos}

En la Argentina, Vicia magellanica se distribuye desde el norte de Mendoza hasta Tierra del Fuego (Correa 1984). Es una especie endémica de esta región. En general, se encuentra asociada, en el norte de la Patagonia, a bosques de transición dominados principalmente por Austrocedrus chilensis (Ezcurra and Brion 2005). Posee raíces pequeñas y rizomas delgados de hasta $50 \mathrm{~cm}$ de largo, algo sub-leñosos. Sus tallos pubescentes pueden medir hasta 65 $\mathrm{cm}$ y sus numerosos zarcillos pueden ser simples o ramosos. Florece desde noviembre hasta marzo; sus flores se disponen en racimos (entre 1 y 4 flores, raramente 7). Las legumbres portan, en general, entre 2 y 6 semillas que suelen medir entre 2-3.5 × 2.1 -3 mm y 2-2.5 $\mathrm{mm}$ de espesor (Correa 1984).

\section{Ensayo de germinación}

Durante el verano de 2012-2013 se recolectaron semillas de una cohorte cultivada en jardín, y provenientes de más de 25 individuos. Las semillas fueron almacenadas en bolsas de papel a temperatura ambiente y mantenidas en oscuridad hasta el comienzo de los experimentos en septiembre de 2013. Para llevar a cabo los ensayos se seleccionaron semillas que no presentaran signos de herbivoría y, en apariencia, sanas, además de evaluar su viabilidad con el método por presión (Sawma and Mohler 2002). Las semillas de $V$. magellanica poseen una cubierta externa dura, como muchas otras especies del género Vicia spp. (Qasem 2020). Utilizando una balanza de precisión (0.001 g) y un calibre digital se registró el peso y el diámetro medio, respectivamente, considerando el diámetro mayor de cada semilla, de 20 semillas seleccionadas aleatoriamente del conjunto de semillas seleccionadas para el ensayo.

Las semillas se sometieron a siete tratamientos simulando efectos directos e indirectos asociados a los incendios y que, según la 
literatura, pueden afectar la germinación de las semillas de distintos grupos taxonómicos (Keeley and Fotheringham 2000). Además, los tratamientos estudiados se seleccionaron con el fin de explorar potenciales alternativas para optimizar la germinación en vistas de utilizar la especie en planes de restauración. Las semillas para cada tratamiento se dispusieron en placas de Petri de $9 \mathrm{~cm}$ de diámetro con sustrato de vermiculita cubierto por papel de filtro. En cada placa se sembraron 40 semillas. Para cada tratamiento, incluido el control, se realizaron cinco repeticiones (i.e., placa de Petri). Los tratamientos fueron los siguientes: 1) control: semillas sin tratamiento asociado al fuego, 2) cenizas continuo: se regaron las semillas durante todo el ensayo de germinación con una solución de $10 \mathrm{~g} / \mathrm{L}$ $(\mathrm{pH}=9.0)$ de cenizas, 3) ceniza inicial: al inicio del ensayo se aplicó a cada placa de Petri 0.5 g de cenizas y el riego a lo largo del ensayo se realizó con agua destilada, 4) $100{ }^{\circ} \mathrm{C}$ : antes de la siembra, las semillas fueron sometidas a calor seco en una estufa con temperatura constante de $100{ }^{\circ} \mathrm{C}$ durante cinco minutos, 5) $120{ }^{\circ} \mathrm{C}$ : antes de la siembra, las semillas fueron sometidas a calor seco en una estufa con temperatura constante de $120^{\circ} \mathrm{C}$ durante 5 minutos, 6) escarificación física: las semillas se escarificaron con papel de lija previo a la siembra, y 7) frío: durante todo el ensayo de germinación, las placas fueron colocadas en heladera a $4{ }^{\circ} \mathrm{C}$ constante con humedad. Las cenizas para los tratamientos de cenizas se obtuvieron a partir de la quema controlada en jardín común de restos leñosos de especies abundantes típicas del bosque nativo de transición de la Patagonia norte; entre ellas, A. chilensis, Lomatia hirsuta, Schinus patagonicus, Maytenus boaria y Nothofagus antarctica. En el caso de la solución, se diluyeron $10 \mathrm{~g}$ de las cenizas obtenidas por cada litro de agua utilizado para el riego, mientras que para el tratamiento de ceniza inicial, $0.5 \mathrm{~g}$ de cenizas obtenidas a partir de la misma quema fueron colocados al inicio del experimento en cada placa de Petri. Las temperaturas asociadas a los tratamientos de calor se encuentran dentro del rango de temperaturas registradas en las capas superficiales del suelo durante la ocurrencia de incendios en bosques y matorrales (Muñoz and Fuentes 1989; Gómez-González and Cavieres 2009; GómezGonzález et al. 2011; Cavallero and Blackhall 2020). Las semillas fueron expuestas a calor seco, ya que la temporada de incendios ocurre durante la estación seca de verano (Franzese et al. 2009).
Durante el período del ensayo, las semillas se mantuvieron en condiciones controladas de laboratorio, en oscuridad y a temperatura constante de $20{ }^{\circ} \mathrm{C}$, salvo las semillas con tratamiento de frío. Debido a que no existe información previa para la especie, a que los requerimientos de luz durante la germinación pueden variar según la temperatura aplicada y a que las semillas almacenadas durante algunos meses a temperatura ambiente pueden perder totalmente o parcialmente la necesidad de luz para lograr germinar (Baskin and Baskin 1998) se decidió para esta primera descripción de la autoecología germinativa de $V$. magellanica unificar la variable luz para todos los tratamientos (i.e., oscuridad). Para mantener la humedad constante se realizó un riego periódico con agua destilada o bien con la solución de cenizas para el tratamiento de cenizas continuo. En caso de aparición de hongos en las repeticiones se registró esta información para cada tratamiento, incluyendo aquellas semillas que germinaron a pesar de encontrarse afectadas. Los hongos registrados durante el ensayo fueron determinados a nivel de género según Hanlin (1998). Desde el inicio del ensayo y durante seis semanas se realizó el conteo de semillas germinadas tres veces por semana, considerando una semilla como germinada al observar la emergencia de la radícula (Baskin and Baskin 1998). Cuando todos los tratamientos de germinación presentaron semillas germinadas, se seleccionaron cinco plántulas de condiciones similares de cada tratamiento (radícula de 1-2 cm, expansión de cotiledones y presencia de primordios de primeras hojas) y se incorporaron al ensayo de crecimiento.

\section{Ensayo de crecimiento}

Para evaluar el desempeño de las plantas creciendo en diferentes condiciones asociadas a posibles escenarios de restauración postfuego se estudió el crecimiento, la floración y la fructificación de plantas creciendo en suelo quemado con y sin aplicación de compost. Para ello, en primera instancia, se extrajo suelo (0-15 cm de profundidad) de diversos puntos de un bosque de Nothofagus pumilio quemado en febrero de 2013. A partir de este sustrato se prepararon los dos tratamientos: suelo quemado sin compost y suelo quemado con un agregado de compost en proporción 7:3. El compost utilizado fue producido en la planta de compostaje de San Carlos de Bariloche a partir de biosólidos urbanos, con mezcla 
de viruta y residuos de poda como agentes estructurantes. Las plantas provenientes del ensayo de germinación se colocaron en macetas individuales (capacidad: $1 \mathrm{~L}$ ), y se registró la fecha de plantación. De cada tratamiento de germinación se trasplantaron cinco individuos (excepto del tratamiento de $120^{\circ} \mathrm{C}$ ), lo que hizo un total de 30 plántulas creciendo en cada tipo de tratamiento: quemado sin compost y quemado con compost.

Las repeticiones (macetas) se mantuvieron en cámara de crecimiento con ciclo de luz: oscuridad de 16:8 horas y con una temperatura que oscilaba entre 16 y $20^{\circ} \mathrm{C}$. El riego se realizó tres veces por semana durante todo el ensayo, excepto durante la última semana cuando se suspendió para simular un evento de sequía. Para ajustar el riego durante el experimento, al inicio del ensayo se pesó el suelo de cinco macetas con suelo quemado sin compost y cinco con compost. Se regaron hasta saturar, se dejaron drenar por $24 \mathrm{~h}$ y luego se volvieron a pesar. Se consideró que entonces el suelo estaba a capacidad de campo. Se estimó el peso de la maceta con el suelo a capacidad de campo y en función de esto se corrigió la humedad una vez por semana tomando los datos de peso de cinco macetas de cada tratamiento. Los demás días el riego se realizó en función de observaciones del estado del suelo. A las siete semanas se registró para cada planta el número de flores y frutos, así como su vitalidad (i.e., aspecto general considerando visualmente la proporción de parte aérea seca) distinguiendo en tres categorías: verde $(0-25 \%$ de biomasa aérea seca), intermedio (25-75\% de biomasa aérea seca) o seco (75-100\% de biomasa aérea seca). Una vez finalizado el ensayo se determinó el peso seco de la parte aérea (incluyendo tallo, hojas, flores y frutos) de cada individuo, y el peso seco de la parte radical de 12 plantas por cada tratamiento. Todo el material fue secado durante $48 \mathrm{~h}$ en estufa a $70{ }^{\circ} \mathrm{C}$.

\section{Análisis de datos}

A partir de los datos obtenidos en los ensayos de germinación se calculó para cada tratamiento el porcentaje de germinación total promedio, el tiempo de latencia promedio, el porcentaje de germinación acumulada a lo largo del ensayo y la tasa de germinación promedio (González-Zertuche and OrozcoSegovia 1996). Además, se calculó el tiempo medio de germinación (TMG) teniendo en cuenta la fórmula 1 (adaptada de Franzese and Ghermandi 2012),

$$
\mathrm{TMG}(\text { días })=\left(\sum t_{i} n_{i}\right) /\left(\sum n_{t}\right)
$$

donde $t_{i}$ es el número de días transcurridos desde la siembra hasta el momento i, y $n_{\text {. }}$ es el número de semillas germinadas en el momento i (cada día). Mediante ANOVA y pruebas a posteriori de Dunnet se evaluaron las posibles diferencias entre los tratamientos y el control tanto para los porcentajes de germinación promedio, el tiempo de latencia y el TMG. Para el cálculo del tiempo de latencia y del TMG no se consideraron los tratamientos que pudieran presentar germinación nula. El porcentaje de semillas afectadas por hongos al final del experimento se analizó mediante una prueba de Kruskal-Wallis y comparaciones múltiples a posteriori.

La biomasa aérea, la biomasa radicular y la biomasa aérea/biomasa radicular fueron analizadas mediante ANOVA para evaluar posibles diferencias entre tratamientos asociados al suelo. Mediante ANCOVA se analizaron las posibles diferencias entre tratamientos en el número de flores y número de frutos, considerando la biomasa seca de la parte aérea como covariable. La vitalidad de las plantas se analizó mediante una prueba de independencia Chi cuadrado.

La normalidad de los residuales fue evaluada mediante pruebas de Shapiro-Wilk y la homogeneidad de variancias mediante pruebas de Levene. Cuando fue necesario, se realizaron transformaciones logarítmicas para optimizar normalidad y homogeneidad de variancias. Todos los análisis se realizaron utilizando el paquete estadístico INFOSTAT@ (versión 2011, FCA, Universidad Nacional de Córdoba, Argentina).

\section{Resultados}

\section{Germinación}

Las semillas presentaron en promedio un peso de $0.029 \pm 0.003 \mathrm{~g}$ y un diámetro medio de $3.42 \pm 0.21 \mathrm{~mm}$. Los resultados mostraron una alta germinación de $V$. magellanica en el tratamiento control (93.5\%) (Figura 1a). Los porcentajes de germinación total promedio de los tratamientos de cenizas continuo, ceniza inicial, escarificación física y frío fueron similares al tratamiento control; variaron entre 90\% y 95\%. Los tratamientos asociados al calor $\left(100{ }^{\circ} \mathrm{C}\right.$ y $\left.120^{\circ} \mathrm{C}\right)$ presentaron menores valores de germinación promedio con respecto a los demás tratamientos $\left(F_{6,28}=138.27, P<0.001\right)$, y la 

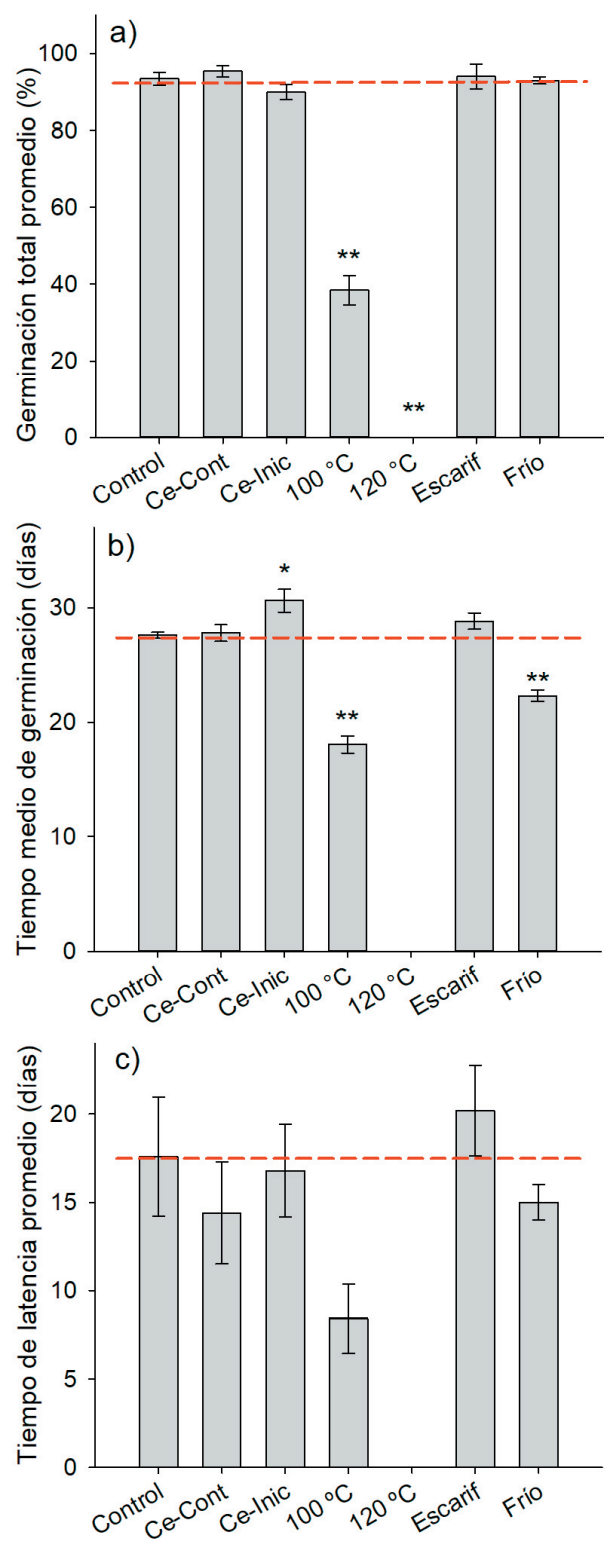

Figura 1. Germinación de Vicia magellanica. a) Germinación total promedio (\%) al finalizar el ensayo; b) Tiempo medio de germinación (días; TMG); c) Tiempo de latencia promedio (días). Los resultados se indican para el control y cada uno de los tratamientos: cenizas continuo (Ce-Cont), ceniza inicial (Ce-Inic), $100^{\circ} \mathrm{C}, 120^{\circ} \mathrm{C}$, escarificación física y frío. Para a) y b), las barras indican terror estándar; prueba a posteriori de Dunnet: ${ }^{*} P<0.05$ $y{ }^{* *} P<0.01$, indicando diferencias significativas entre tratamientos y control (control: línea punteada).

Figure 1. Germination of Vicia magellanica. a) Mean total germination (\%) at the end of the experiment; b) Mean germination time (days; TMG); c) Mean germination latency (days). Results are described for the control and each treatment: continued ash (Ce-Cont), initial ash (Ce-Inic), $100{ }^{\circ} \mathrm{C}, 120{ }^{\circ} \mathrm{C}$, physical scarification, and cold. For a) and b), bars indicate \pm standard error; Dunnet post-hoc test: ${ }^{*} P<0.05$ and ${ }^{* *} P<0.01$, indicating significant differences between treatments and control (control: dashed line). germinación a $120^{\circ} \mathrm{C}$ fue nula (Dunnet: $P<0.01$ para ambos tratamientos de calor).

El TMG de las semillas de $V$. magellanica difirió según el tratamiento aplicado $\left(F_{5,24}=46.46\right.$, $P<0.0001)$ (Figura 1b). Las semillas sometidas al tratamiento de ceniza inicial registraron un TMG 11\% mayor en comparación al control (Dunnet, $P<0.01)$. Las semillas sometidas a tratamientos de temperatura (calor y frío) mostraron un TMG significativamente menor al observado en el tratamiento control (Dunnet, $P<0.01)$. En particular, el tratamiento de $100{ }^{\circ} \mathrm{C}$ presentó una tasa de germinación sin máximos pronunciados o picos y un menor tiempo de latencia promedio en comparación con los demás tratamientos (marginalmente significativo, $F_{5,24}=2.52$, $P=0.057$ ) (Figura 1c; Figura 2). En general, las curvas de germinación acumulada y la tasa de germinación para los diversos tratamientos, excepto los de aplicación de calor, muestran que la germinación se inició con un pulso abrupto y marcado, alcanzando los valores máximos aproximadamente a la semana del registro de inicio de la germinación (Figura 2).

En general, los porcentajes de semillas atacadas por hongos fueron bajos y similares para los tratamientos de cenizas (continuo e inicial), escarificación, frío y control, mostrando valores que variaron entre $0-6 \%$ de semillas afectadas. Las semillas sometidas a calor fueron significativamente más afectadas por hongos (más del 75\%), en comparación a los demás tratamientos $(H=25.20, P<0.001$; comparaciones múltiples: $P<0.01$ para ambos tratamientos de calor). Dentro de los hongos atacantes se identificaron especies pertenecientes a los géneros Rhizopus spp., Aspergillus spp., Penicillium spp. y Stachybotrys spp.

\section{Crecimiento}

Tanto la biomasa aérea como la biomasa radicular fueron significativamente mayores en las plantas creciendo en los tratamientos de suelo quemado sin compost, en comparación con las plantas creciendo en suelo con adición de compost (biomasa aérea: $F_{1,56}=29.28, P<0.001$; biomasa radicular: $F_{1,22}=94.60, P<0.001$ ) (Figura 3a). La proporción de biomasa aérea/biomasa radicular también difirió entre tratamientos; fue cinco veces mayor para los individuos creciendo en suelo quemado con compost, en comparación con los individuos creciendo sin compost $\left(F_{1,22}=55.96, P<0.001\right)$ (Figura $\left.3 b\right)$. 
a)

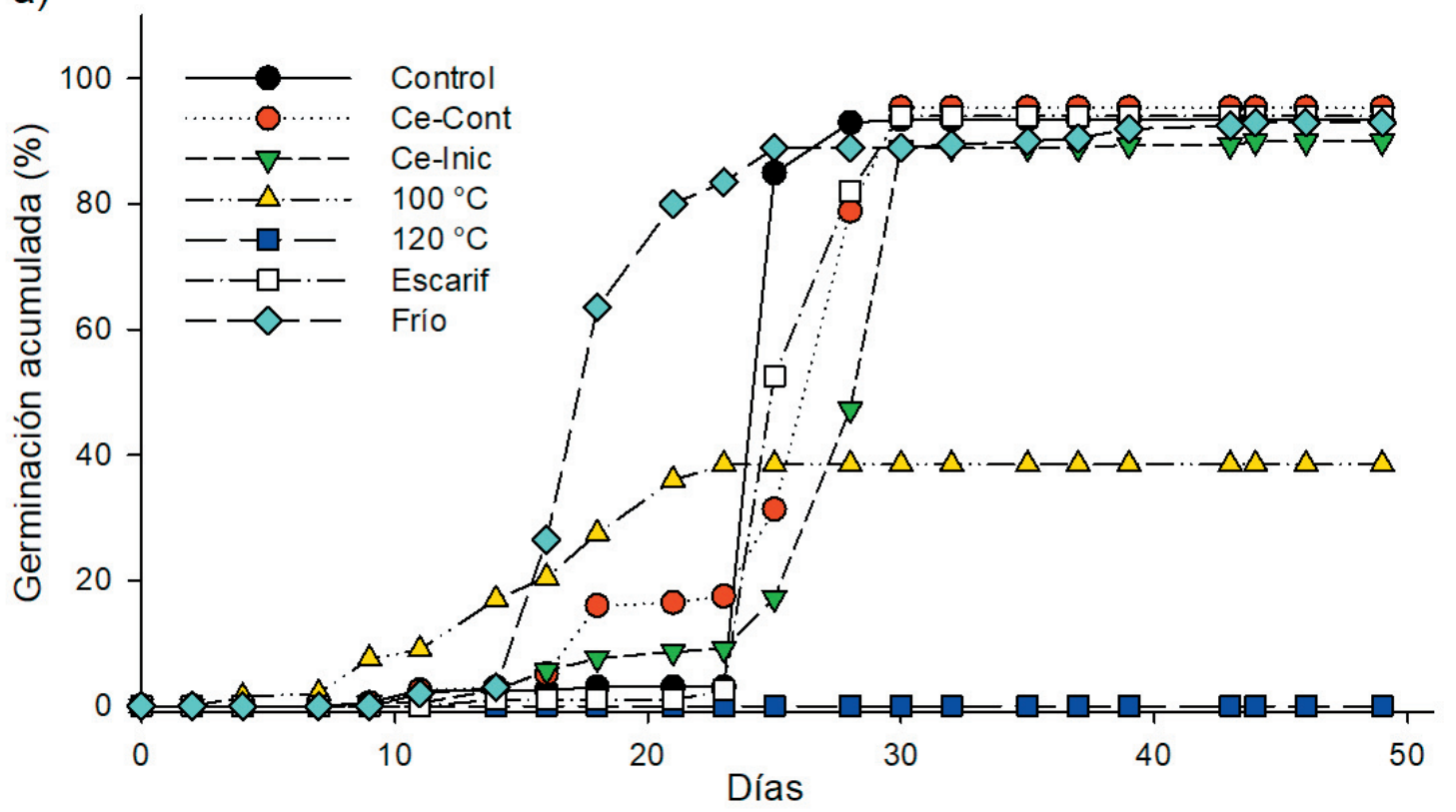

b)

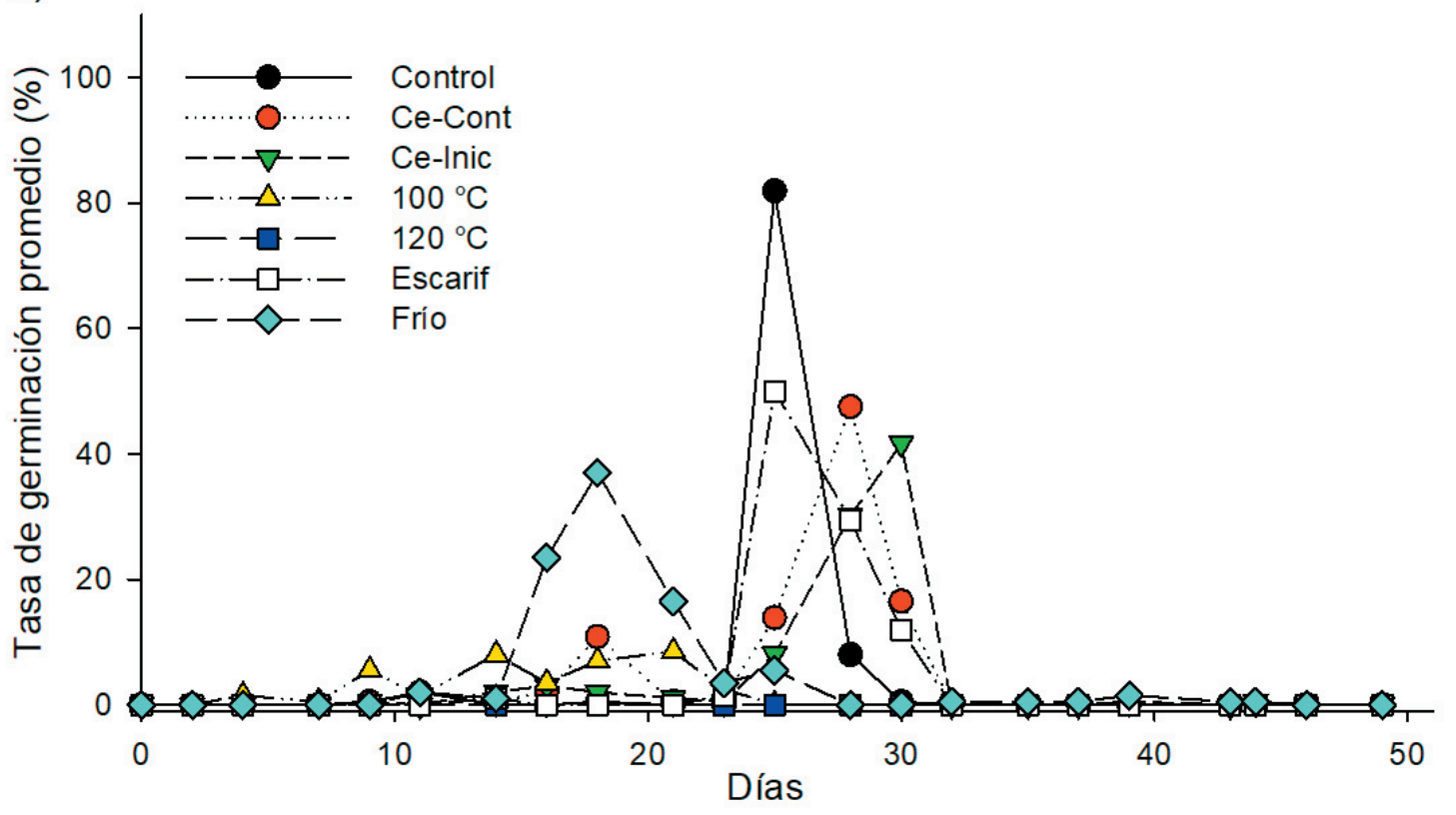

Figura 2. Germinación de Vicia magellanica. a) Germinación acumulada (\%) a lo largo del ensayo; b) Tasa de germinación promedio (\%). Los resultados se indican para el control y cada uno de los tratamientos: cenizas continuo (Ce-Cont), ceniza inicial (Ce-Inic), $100{ }^{\circ} \mathrm{C}, 120^{\circ} \mathrm{C}$, escarificación física y frío.

Figure 2. Germination of Vicia magellanica. a) Cumulative germination (\%) across the complete experiment; b) Mean germination rate $(\%)$. Results are described for the control and each treatment: continued ash (Ce-Cont), initial ash (Ce-Inic), $100^{\circ} \mathrm{C}, 120^{\circ} \mathrm{C}$, physical scarification, and cold. 



Figura 3. Crecimiento de Vicia magellanica bajo dos tratamientos de suelo: suelo quemado sin compost y suelo quemado con compost. a) Biomasa seca (g) radicular y aérea; b) Proporción de biomasa aérea/biomasa radicular; c) Número de plantas que presentaron diferente vitalidad luego de la simulación de sequía (verde, intermedio, seco; ver texto para detalles sobre la clasificación). Las barras indican terror estándar. Letras diferentes indican diferencias significativas $(P<0.05)$ entre los dos tratamientos.

Figure 3. Growth of Vicia magellanica under two soil treatments: burned soil without compost and burned soil with compost. a) Root and aerial dry biomass (g); b) Aerial biomass/root biomass proportion; c) Number of plants that showed different vitality after the simulated drought (green, intermediate, dry; see text for classification details). Bars indicate \pm standard error. Different letters indicate significant differences $(P<0.05)$ between treatments.
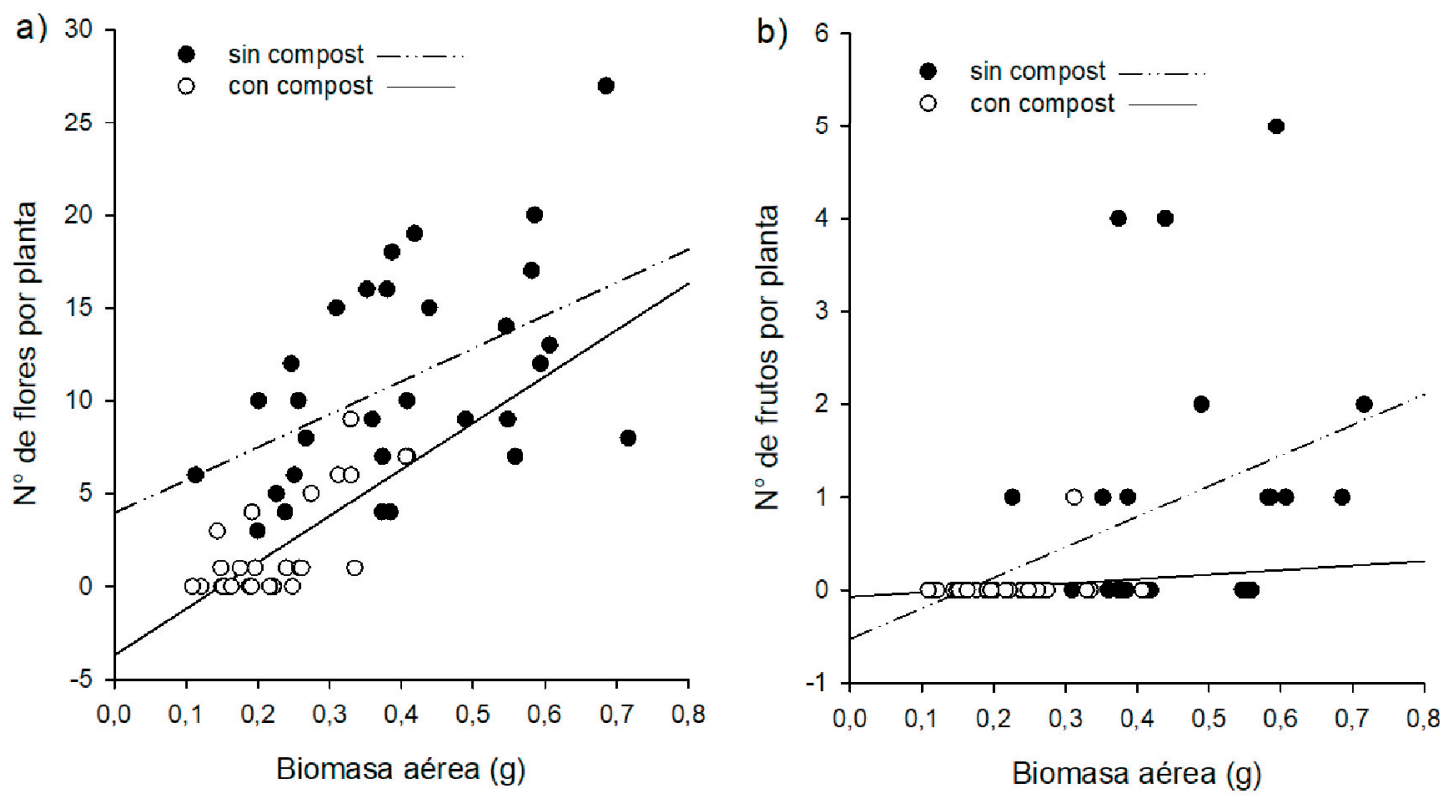

Figura 4. Número de a) Flores y b) Frutos por planta de Vicia magellanica, en relación con la biomasa aérea (g) de cada individuo, para los dos tratamientos de suelo aplicados en el ensayo de crecimiento (suelo quemado sin compost y con compost). Las líneas muestran la correlación lineal entre la biomasa área y la variable respuesta.

Figure 4. Number of a) Flowers and b) Fruits per plant of Vicia magellanica, in relation to the aerial biomass (g) of each individual, for the two soil treatments in the growth experiment (burned soil with and without compost). Lines show the lineal correlation between aerial biomass and the response variable. 
Luego de la sequía simulada, al final del ensayo de crecimiento, las plantas creciendo en los dos tratamientos de suelo mostraron diferente vitalidad $\left(\chi^{2}\right.$ de Pearson $=41.14$, $P<0.001$ ) (Figura 3c). El 93\% de las plantas creciendo en suelo quemado sin compost mostraron un aspecto verde y vigoroso, y además, saludable (i.e., sin daños aparentes), mientras que sólo $11 \%$ de las plantas en suelo quemado con compost fueron clasificadas en esta categoría de apariencia.

El número de flores y el número de frutos por planta se correlacionó significativamente con la biomasa aérea (flores: $F_{1,55}=22.5, P<0.001$, coeficiente de regresión lineal=19.18; frutos: $F_{1,55}=10.76, P<0.01$, coeficiente de regresión lineal=1.13). Luego de siete semanas, el número de flores fue significativamente mayor en las plantas creciendo en suelo quemado sin compost $\left(F_{1,55}=21.53, P<0.001\right)$ (Figura $\left.4 a\right)$, mientras que no se encontraron diferencias significativas en el número de frutos entre las plantas creciendo en distintos tratamientos de suelo $\left(F_{1,55}=1.03, P=0.31\right)$ (Figura $4 \mathrm{~b}$ ).

\section{Discusión}

Los resultados obtenidos en este estudio aportan nueva información sobre la autoecología de $V$. magellanica, dado que no existen registros previos de estudios que hayan evaluado la germinación de esta especie endémica. En general, los porcentajes de germinación fueron muy altos $(>90 \%$ de germinación total promedio en todos los tratamientos), excepto en los asociados a la aplicación de calor previo. Luego de unos meses de conservación de las semillas en oscuridad, a temperatura constante $\left(20^{\circ} \mathrm{C}\right)$ y sin humedad, las semillas de $V$. magellanica en el tratamiento control presentaron un alto porcentaje de germinación. Además, los diversos tratamientos asociados a presencia de cenizas, cambio de $\mathrm{pH}$ en suelo, escarificación física y temperaturas frías prolongadas, no afectaron su germinación. El calor seco, asociado a las altas temperaturas durante un incendio, posee un efecto negativo sobre la germinación de $V$. magellanica.

El alto porcentaje de germinación registrado en el control es particularmente notorio, ya que son numerosas las especies de leguminosas que poseen una testa externa dura al igual que $V$. magellanica y necesitan de algún mecanismo (físico o químico) para interrumpir la dormancia (Smýkal et al. 2014).
Por ejemplo, se determinó que V. nigricans, cuyas semillas también poseen una cubierta externa dura y es una especie nativa que en la región de estudio, puede registrarse en los mismos sitios post-fuego que $V$. magellanica, presenta bajos porcentajes de germinación en condiciones control $(7 \%)$, y este porcentaje puede triplicarse luego de aplicar calor seco $\left(120^{\circ} \mathrm{C}\right)$ y ceniza (Blackhall et al. 2016). Por el contrario, los resultados del presente trabajo muestran patrones similares a los observados para especies de Vicia spp. en China, creciendo en zonas también subalpinas y de clima templado, donde se han determinado porcentajes de germinación muy elevada a $20^{\circ} \mathrm{C}$, además de a bajas temperaturas $\left(5^{\circ} \mathrm{C}\right)$ (Hu et al. 2013).

En general, la escarificación física y química (en particular, cenizas y, sobre todo, el tratamiento de cenizas continuo) asociadas al ambiente post-fuego, no influyeron ni sobre el porcentaje de germinación ni en el tiempo de latencia ni sobre el tiempo de germinación media. En contraste, ambos tratamientos de temperatura para los que sí se observaron semillas germinadas $\left(4^{\circ} \mathrm{C}\right.$ y $100{ }^{\circ} \mathrm{C}$ ) disminuyeron el tiempo medio de germinación y la misma tendencia se observó para el tiempo de latencia, aunque sólo a $4^{\circ} \mathrm{C}$ se registraron valores muy altos de germinación total. Los resultados indicarían que la estratificación fría de las semillas tiene un efecto directo sobre el inicio del proceso de germinación y sobre la anticipación en el pico de la tasa de germinación, pero sin afectar el porcentaje final de semillas germinadas. Este hecho podría relacionarse con la capacidad de una especie de iniciar el repoblamiento de un área quemada (especies pioneras), en donde, en asociación con una menor cobertura vegetal, es común a nivel de micrositio registrar temperaturas más bajas y mayores amplitudes térmicas sobre el suelo en invierno y primavera, en comparación con micrositios de áreas no quemadas (Blackhall et al. 2015). Este proceso podría replicarse también en micrositios de áreas abiertas con ausencia de dosel, no necesariamente asociadas a sitios post-fuego. Además, en el contexto del clima mediterráneo del noroeste de la Patagonia (inviernos húmedos y fríos, y veranos muy secos y cálidos), esta capacidad de tolerar temperaturas bajas durante el proceso de germinación le permitiría a las semillas de $V$. magellanica iniciar este proceso cuando aún hay suficiente humedad en el suelo, y formar raíces profundas antes de la llegada 
de la estación seca, que comienza con el inicio del verano.

La ausencia de germinación a $120^{\circ} \mathrm{C}$ indicaría que la exposición durante al menos 5 minutos a temperaturas $\geq 120{ }^{\circ} \mathrm{C}$ anula la capacidad germinativa de las semillas. De forma similar, en un estudio en el este de Australia sobre la germinación de 35 especies de leguminosas que presentan semillas con cubierta externa dura y tamaños muy variables, se encontró que la mayoría de las especies no lograron germinar cuando fueron sometidas a $120{ }^{\circ} \mathrm{C}$ por lapsos mayores a 1 minuto. Sin embargo, y en contraposición a lo registrado para $V$. magellanica, para la mayoría de las especies australianas se observó una estimulación de la germinación a $100{ }^{\circ} \mathrm{C}$, incluyendo diversos lapsos de exposición al calor (Auld and O'Connell 1991). Por otro lado, en el presente trabajo se observó que las semillas sometidas a ambos tratamientos de calor presentaron una mayor incidencia de hongos durante el ensayo. Todos los hongos determinados corresponden a géneros degradadores de azúcares simples, capaces de colonizar las semillas y consumir sustancias a través de la cutícula (Alexopoulos et al. 1996). Esto explicaría la mayor cantidad de semillas afectadas por hongos en los tratamientos a $100{ }^{\circ} \mathrm{C}$ y $120{ }^{\circ} \mathrm{C}$ con respecto a los demás tratamientos, ya que el calor debilitaría fuertemente la cubierta externa. No obstante, se pudo constatar que varias de las semillas sometidas a $100{ }^{\circ} \mathrm{C}$ y atacadas por hongos, conservaron su capacidad de germinar.

Teniendo en cuenta la baja tolerancia de las semillas a la exposición de calor asociado al fuego, la ocurrencia de incendios muy intensos sería una limitante para la aparición de esta especie respecto a otras en el banco de semillas viables. Estos datos resultarían inesperados para una especie que a menudo fue relevada en sitios con incendios recientes (e.g., Urretavizcaya et al. 2018). Algunos factores como la capacidad para enterrarse y la formación de un banco de semillas persistente se asociaron con la germinación post-fuego de especies (Keeley and Fotheringham 2000). En este sentido, las semillas de V. magellanica tienen un tamaño relativamente grande con respecto a otras leguminosas (Auld and O'Connell 1991; Hanley et al. 2003; Van Assche et al. 2003), lo que no facilitaría un enterramiento rápido muy profundo (Baskin and Baskin 1998). Estudios aún no publicados por nuestro grupo de trabajo indican que $V$. magellanica mantiene su potencial germinativo por mucho tiempo (>10 años). Esta ventana de viabilidad a lo largo de los años podría favorecer su enterramiento parcial a pesar de su tamaño; además, ante la ocurrencia de incendios que consuman la sección más superficial de la hojarasca y materia orgánica del suelo, las semillas grandes podrían germinar desde una mayor profundidad, donde, adicionalmente, el efecto de la temperatura del fuego es menor. Por ejemplo, Hanley et al. (2003) registraron para diversas especies de leguminosas que las semillas de mayor tamaño presentaban una mayor mortalidad a altas temperaturas, incluyendo tratamientos a $120^{\circ} \mathrm{C}$, y que las semillas de estas especies suelen beneficiarse de su capacidad de emerger desde una mayor profundidad en el suelo en comparación con las especies que poseen semillas más pequeñas.

Por otro lado, el efecto del calor del fuego sobre el suelo a nivel de micrositio es muy heterogéneo, ya que la temperatura del perfil y la profundidad a la que se observa el efecto varía según la influencia del contenido de agua edáfica, la duración de la exposición y las propiedades físicas del suelo (Albanesi and Anriquez 2003). Incluso, pequeñas variaciones en la cantidad y el tipo de combustible afectan a microescala las temperaturas máximas alcanzadas y los lapsos que perduran estas temperaturas en el suelo, lo que crea una heterogeneidad compleja de micrositios en donde las diversas semillas del banco pueden verse beneficiadas o perjudicadas (Wiggers et al. 2013). No se cuenta con datos concretos y específicos sobre temperaturas reales del perfil del suelo durante incendios en bosques y matorrales del noroeste patagónico. Sin embargo, registros de zonas ecológicamente similares y la extrapolación de datos de carga de combustible en la región indicarían que las temperaturas del suelo pueden variar entre 40 y $140{ }^{\circ} \mathrm{C}$ (entre los 2 a $10 \mathrm{~cm}$ de profundidad del suelo) (Muñoz and Fuentes 1989; Kunst and Bravo 2003; Gómez-González and Cavieres 2009; Blackhall 2012), rango sobre el cual, a futuro, podría continuar la exploración de su capacidad de germinación. En síntesis, el patrón observado de regeneración post-fuego de V. magellanica podría estar determinado por la heterogeneidad intrínseca de la severidad del fuego a nivel de micrositio, además de su capacidad de germinar rápidamente, aunque sea en forma reducida, al ser las semillas expuestas a temperaturas moderadas.

Los resultados del ensayo de crecimiento en el que se evaluó el desempeño de $V$. magellanica 
en un posible escenario de restauración activa luego de un incendio mostraron que las condiciones edáficas asociadas a un suelo quemado permitieron un desarrollo satisfactorio. En suelos quemados sin adición de compost se observó una mayor biomasa aérea y radicular, y un mayor número de flores en comparación con las plantas creciendo con agregado de compost. Estas características le supondrían una ventaja para colonizar áreas con suelos degradados. En diferentes tipos de ecosistemas y considerando diversas situaciones asociadas a restauración postdisturbio (e.g., erosión hídrica, contaminación por oleoductos, incendios), numerosos trabajos determinaron que el agregado de compost orgánico, incluyendo compost de biosólidos, puede aumentar la biomasa o cobertura vegetal a escala de comunidad (e.g., Guerrero et al. 2001; Meyer et al. 2001; Martínez et al. 2003; Walter and Calvo 2009; Kowaljow et al. 2010; Larney and Angers 2012; Ferreiro et al. 2020). Sin embargo, en algunos estudios en los que se evaluó el desempeño específico de determinadas especies vegetales con la aplicación de diferentes tipos de fertilizantes se observó que las respuestas de las plantas pueden ser tanto positivas como negativas, y esto depende de la especie de estudio, del tipo de fertilizante utilizado y de la concentración del mismo. Por ejemplo, en algunas especies del genero Vicia spp., como $V$. faba y $V$. sativa, el agregado de diferentes concentraciones de compost de biosólidos incrementa el crecimiento y la productividad de los cultivos (Fernández-Luqueño et al. 2012; Saruhan et al. 2015; Zendejas et al. 2015). En contraposición, Tang et al. (2014) determinaron también para $V$. sativa que el agregado de diferentes concentraciones y combinaciones de nutrientes (N, P o K) puede en algunos casos disminuir y en otros aumentar la densidad de individuos y su crecimiento en comparación a los tratamientos control sin agregado de fertilizantes.

En la región de estudio y aplicando compost de biosólidos del mismo origen que en el presente trabajo también se observó que la aplicación de enmienda puede reducir inicialmente la supervivencia de plántulas de A. chilensis y Nothofagus dombeyi, dos especies arbóreas dominantes de los bosques en donde habita V. magellanica (Gobbi and Labud 2005), y esta disminución fue asociada al aumento en la conductividad eléctrica en niveles muy superiores a los del suelo sin agregado de compost. Por otro lado, Nursu'aidah (2014) ha registrado para la leguminosa de cultivo Vigna unguiculata sesquipedalis que aplicar fertilizantes puede reducir la eficiencia en la fijación de nitrógeno al inducir una menor producción de nódulos en las plantas y, a su vez, lo ha relacionado directamente a una menor producción de biomasa. Nuestros resultados muestran que el agregado de enmienda orgánica en $V$. magellanica no posee un efecto positivo en su crecimiento, como sí se ha observado para otras especies y comunidades. Los resultados de este trabajo indicarían que esta especie puede desarrollarse con éxito en suelos recientemente quemados, sin agregado de nutrientes. Además, la menor relación biomasa aérea/biomasa radicular observada en plantas sin adición de compost podría conferirle a la especie propiedades esenciales para enfrentar la época estival con valores de humedad en el suelo menores al 5\% (Varela et al. 2011; de Paz 2014), como fue observado experimentalmente y de forma indirecta en la simulación de sequía al final del ensayo. Sin embargo, sería aconsejable a futuro explorar el efecto de la aplicación de diferentes proporciones de compost de biosólidos sobre el desempeño de $V$. magellanica, como así también otros tipos de enmiendas, el efecto de las mismas sobre los nódulos radiculares que fueron observados en las plantas, las condiciones edáficas de los suelos o la potencial variabilidad asociada al uso de semillas de una única cohorte en contraste a otras fuentes de semillas.

La producción de flores fue mayor en suelos degradados, y aunque no se pudo probar que la producción de frutos siga el mismo patrón y que harían falta otros experimentos para comprobarlo, es muy posible que la fertilidad de $V$. magellanica sea mayor en suelos quemados. De ser así, y teniendo en cuenta su capacidad de florecer y fructificar en pocos meses luego de su germinación, con pocas siembras podría establecerse una población de la especie que persista en el tiempo en el marco de un proyecto de restauración, reduciendo en gran medida los esfuerzos económicos y de trabajo asociados con la revegetación.

En síntesis, V. magellanica presenta numerosas características que serían destacables para su uso en estrategias de revegetación postfuego en bosques y matorrales del noroeste patagónico aplicando siembra directa a campo, y particularmente en los primeros estadios luego del incendio. La presencia de cenizas y la escarificación física no afectan el porcentaje 
final de germinación, en comparación con alto porcentaje de germinación del tratamiento control. Además, la estratificación por frío, factor ambiental que es más intenso en zonas post-fuego sin vegetación, fue el tratamiento que estimuló en mayor medida la germinación temprana, manteniendo un elevado porcentaje de germinación. Por otro lado, el hecho de que los individuos creciendo en suelos quemados sin compost se desarrollen exitosamente y que, además, se registre un mayor porcentaje de vigorosidad ante situaciones de sequía estival (en comparación con lo observado en suelos quemados con compost) haría suponer que en aquellos lugares donde $V$. magellanica fuera sembrada como medida de revegetación no sería necesario añadir enmiendas orgánicas al suelo. Si bien la aparición de especies en escenarios post-fuego depende en primera instancia de la capacidad de germinación o de rebrote de las mismas, las plantas deben superar otros factores limitantes asociados al establecimiento y a su crecimiento. En este sentido, la asociación de $V$. magellanica con microorganismos fijadores de $N$ (nutriente limitante en bosques post-fuego del noroeste patagónico) (Alauzis et al. 2004; Varela et al. 2011) podría conferirle una ventaja con respecto a otras especies pioneras. Como ya fuera documentado para otros tipos de ambientes (del Moral and Wood 1993; Wardle and Peltzer 2007), durante la sucesión primaria luego de un disturbio severo, las especies capaces de fijar nitrógeno a través de simbiosis favorecen de manera significativa la restauración de las condiciones del suelo y permiten una rápida recuperación del ambiente.

Agradecimientos. Agradecemos a N. de la Rosa por su colaboración en la determinación micológica en el ensayo de germinación.

\section{ReFERENCIAS}

Alauzis, M. V., M. J. Mazzarino, E. Raffaele, and L. Roselli. 2004. Wildfires in NW Patagonia: long-term effects on a Nothofagus forest soil. Forest Ecology and Management 192:131-142. https://doi.org/10.1016/j.foreco.2003.11.014.

Albanesi, A., and A. Anriquez. 2003. El fuego y el suelo. Pp. 47-59 en C. R. Kunst, S. Bravo y J. L. Panigatti (eds.). Fuego en los Ecosistemas Argentinos. INTA, Santiago del Estero, Argentina.

Alexopoulos, C. J., C. W. Mims, and M. Blackwell. 1996. Introductory mycology. Fourth Edition. John Wiley and Sons, New York, USA.

Auld, T. D., and M. A. O'Connell. 1991. Predicting patterns of post'fire germination in 35 eastern Australian Fabaceae. Australian Journal of Ecology 16:53-70. https://doi.org/10.1111/j.1442-9993.1991.tb01481.x.

Baigorria, T., D. Gómez, C. Cazorla, A. Lardone, M. Bojanich, B. Aimetta, A. Bertolla, M. Cagliero, D. Vilches, D. Rinaudo, and A. Canal. 2013. Capítulo 19: Bases para el manejo de vicia como antecesor del cultivo de maíz. Pp. 158-164 en C. Álvarez, A. Quiroga, D. Santos y M. Bodrero (eds.). Contribuciones de los cultivos de cobertura a la sostenibilidad de los sistemas de producción. INTA, La Pampa, Argentina. https://inta.gob.ar/sites/default/files/scripttmp-inta_cultivos_de_cobertura_.pdf\#page $=158$

Baskin, C. C., and J. M. Baskin. 1998. Seeds: ecology, biogeography and evolution of dormancy and germination. Academic Press, San Diego, USA.

Blackhall, M. 2012. Respuestas de especies leñosas a herbívoros e incendios en bosques y matorrales del noroeste de la Patagonia: Estudio de la inflamabilidad vegetal. Tesis Doctoral. Centro Regional Universitario Bariloche, Universidad Nacional del Comahue, San Carlos de Bariloche. Argentina. Pp. 184.

Blackhall, M., J. Franzese, E. Raffaele, and M. E. Gobbi. 2016. Efecto del fuego sobre la germinación de especies leñosas y trepadoras de los bosques y matorrales del noroeste patagónico. Pp. 445-449 en Libro de Actas V Jornadas Forestales Patagónicas, III Jornadas Forestales de Patagonia Sur y Ecofuego II, Esquel, Chubut, Argentina.

Blackhall, M., E. Raffaele, and T. T. Veblen. 2008. Cattle affect early post-fire regeneration in a Nothofagus dombeyiAustrocedrus chilensis mixed forest in northern Patagonia, Argentina. Biological Conservation 141:2251-2261. https: //doi.org/10.1016/j.biocon.2008.06.016.

Blackhall, M., E. Raffaele, and T. T. Veblen. 2015. Combined effects of fire and cattle in shrublands and forests of northwest Patagonia. Ecología Austral 25:1-10. https://doi.org/10.25260/EA.15.25.1.0.48.

Bond, W. J., and B. W. van Wilgen. 1996. Fire and Plants. Population and Community Biology Series 14. Chapman and Hall, London, UK. https://doi.org/10.1007/978-94-009-1499-5.

Cavallero, L., and M. Blackhall. 2020. Resprouting increases seedling persistence likelihood after fire in a semelparous bamboo species. Acta Oecologica 108:103623. https://doi.org/10.1016/j.actao.2020.103623.

Correa, M. N. 1984. Flora Patagónica. Dicotiledoneae dialipétalas (Salicaceae a Cruciferae). Tomo 8, Parte 4a. Colección Científica INTA, Buenos Aires, Argentina.

Downie, J. A. 2005. Legume haemoglobins: symbiotic nitrogen fixation needs bloody nodules. Current Biology 15: 196-198. https://doi.org/10.1016/j.cub.2005.03.007.

Ezcurra, C., and C. Brion. 2005. Plantas del Nahuel Huapi. Catálogo de la Flora Vascular del Parque Nacional Nahuel Huapi, Argentina. Universidad Nacional del Comahue, Bariloche, Argentina.

Fenner, M., and K. Thompson. 2005. Seed dormancy. Pp. 97-109 in M. Fenner and K. Thompson (eds.). The ecology of seeds. Cambridge University Press, Nueva York, USA. 
Fernández-Luqueño, F., L. Corlay-Chee, E. Robledo-Santoyo, J. Pineda-Pineda, A. Vázquez-Alarcón, L. A. MirandaRomero, G. Cabrera-Lazaro, J. Méndez-Bautista, F. López-Valdez, and L. Dendooven. 2012. Growth and development of common vetch (Vicia sativa L.) in a gasoline-polluted soil amended with organic or inorganic amendments. African Journal of Agricultural Research 7:1259-1267.

Ferreiro, N., P. Satti, M. González'Polo, and M. J. Mazzarino. 2020. Composts promote short'term rehabilitation in a Patagonian roadside affected by tephra deposition. Restoration Ecology 28:73-81. https://doi.org/10.1111/rec.13034.

Franzese, J., and L. Ghermandi. 2012. Effect of fire on recruitment of two dominant perennial grasses with different palatability from semi-arid grasslands of NW Patagonia (Argentina). Plant Ecology 213:471-481.

Franzese, J., L. Ghermandi, and D. Bran. 2009. Post'fire shrub recruitment in a semi'arid grassland: the role of microsites. Journal of Vegetation Science 20:251-259. https://doi.org/10.1111/j.1654-1103.2009.05733.x.

Gobbi, M. E., and V. Labud. 2005. Respuesta de plantines de ciprés (Austrocedrus chilensis) y coihue (Nothofagus dombeyi) a la aplicación de composts. Pp. 83-87 en I Reunión sobre ecología, conservación y uso de los bosques de ciprés de la cordillera. Esquel, Chubut, Argentina.

Gobbi, M. E., J. Puntieri, and S. Calvelo. 1995. Post-fire recovery and invasion by alien plant species in a South American woodland-steppe ecotone. Pp. 105-115 in P. Pysek, K. Prach, M. Rejmanek and M. Wade (eds.). Plant Invasions: General Aspects and Special Problems. Academic Publishing, Amsterdam, Germany.

Gómez Ruiz, P. A. 2011. Efecto de la densidad de siembra sobre las interacciones biológicas entre las leguminosas Lupinus bogotensis y Vicia benghalensis con las nativas Solanum oblongifolium y Viburnum tinoides en parcelas experimentales de restauración ecológica del bosque altoandino. Tesis de Magister. Universidad Nacional de Colombia, Bogotá. Colombia. Pp. 108.

Gómez-González, S., and L. A. Cavieres. 2009. Litter burning does not equally affect seedling emergence of native and alien species of the Mediterranean-type Chilean matorral. International Journal of Wildland Fire 18:213-221. https: //doi.org/10.1071/WF07074.

Gómez-González, S., C. Torres-Díaz, and E. Gianoli. 2011. The effects of fire-related cues on seed germination and viability of Helenium aromaticum (Hook.) HL Bailey (Asteraceae). Gayana Botánica 68:86-88. https://doi.org/10.4067/ S0717-66432011000100007.

González-Zertuche, L., and A. Orozco-Segovia. 1996. Métodos de análisis de datos en la germinación de semillas, un ejemplo: Manfreda brachystachya. Botanical Sciences 58:15-30. https://doi.org/10.17129/botsci.1484.

Guerrero, C., I. Gómez, R. Moral, J. Mataix-Solera, J. Mataix-Beneyto, and T. Hernández. 2001. Reclamation of a burned forest soil with municipal waste compost: macronutrient dynamic and improved vegetation cover recovery. Bioresource Technology 76:221-227. https://doi.org/10.1016/S0960-8524(00)00125-5.

Hanley, M., J. Unna, and B. Darvill. 2003. Seed size and germination response: a relationship for fire-following plant species exposed to thermal shock. Oecologia 134:18-22. https://doi.org/10.1007/s00442-002-1094-2.

Hanlin, R. T. 1998. Combined keys to Illustrated genera of ascomycetes. Volumes I and II. American Phytopathological Society Press, St. Paul, Minnesota, USA.

Hu, X., T. Li, J. Wang, Y. Wang, C. C. Baskin, and J. M. Baskin. 2013. Seed dormancy in four Tibetan Plateau Vicia species and characterization of physiological changes in response of seeds to environmental factors. Seed science research 23:133-140. https://doi.org/10.1017/S0960258513000019.

Keeley, J. E., and C. J. Fotheringham. 2000. Role of fire in regeneration from seed. Pp. 311-330 in M. Fenner (ed.). Seeds: the ecology of regeneration in plant communities. Second Edition. CABI Publishing, New York, New York, USA. https://doi.org/10.1079/9780851994321.0311.

Kitzberger, T., G. L. W. Perry, J. Paritsis, J. H. Gowda, A. J. Tepley, A. Holz, and T. T. Veblen. 2016. Fire-vegetation feedbacks and alternative states: common mechanisms of temperate forest vulnerability to fire in southern South America and New Zealand. New Zealand Journal of Botany 54:247-272. https://doi.org/10.1080/0028825X.2016.1151903.

Kowaljow, E., M. J. Mazzarino, P. Satti, and C. Jiménez-Rodríguez. 2010. Organic and inorganic fertilizer effects on a degraded Patagonian rangeland. Plant and Soil 332:135-145. https://doi.org/10.1007/s11104-009-0279-4.

Kunst, C., and S. Bravo. 2003. Fuego, calor y temperatura. Pp. 39-45 en C. R. Kunst, S. Bravo y J. L. Panigatti (eds.). Fuego en los ecosistemas Argentinos. INTA, Santiago del Estero, Argentina.

Larney, F. J., and D. A. Angers. 2012. The role of organic amendments in soil reclamation: a review. Canadian Journal of Soil Science 92:19-38. https://doi.org/10.4141/cjss2010-064.

van der Maarel, E. 2005. Vegetation ecology-an overview. Pp. 1-51 in E. van der Maarel (ed.). Vegetation ecology. Blackwell, Oxford, UK.

Mariani, M., A. Holz, T. T. Veblen, G. Williamson, M. Fletcher, and D. M. J. S. Bowman. 2018. Climate change amplifications of climate'fire teleconnections in the Southern Hemisphere. Geophysical Research Letters 45:50715081. https://doi.org/10.1029/2018GL078294.

Martínez, F., G. Cuevas, R. Calvo, and I. Walter. 2003. Biowaste effects on soil and native plants in a semiarid ecosystem. Journal of Environmental Quality 32:472-479. https://doi.org/10.2134/jeq2003.4720.

Meyer, V. F., E. F. Redente, K. A. Barbarick, and R. Brobst. 2001. Biosolids applications affect runoff water quality following forest fire. Journal of Environmental Quality 30:1528-1532. https://doi.org/10.2134/jeq2001.3051528x.

del Moral, R., L. R. Walker, and J. P. Bakker. 2007. Insights gained from succession for the restoration of landscape structure and function. Pp. 19-44 in L. R. Walker, J. Walker and R. J. Hobbs (eds.). Linking restoration and ecological succession. Springer, New York, New York, USA. https://doi.org/10.1007/978-0-387-35303-6_2.

del Moral, R., and D. M. Wood. 1993. Early primary succession on the volcano Mount St. Helens. Journal of Vegetation 
Science 4:223-234. https://doi.org/10.2307/3236108.

Morales, D., C. M. Rostagno, and L. La Manna. 2013. Runoff and erosion from volcanic soils affected by fire: the case of Austrocedrus chilensis forests in Patagonia, Argentina. Plant and Soil 370:367-380. https://doi.org/10.1007/s11104013-1640-1.

Mortlock, B. W. 2000. Local seed for revegetation: where will all that seed come from? Ecological Management and Restoration 1:93-101. https://doi.org/10.1046/j.1442-8903.2000.00029.x.

Muñoz, M. R., and E. R. Fuentes. 1989. Does fire induce shrub germination in the Chilean matorral? Oikos 56:177-181. https://doi.org/10.2307/3565333.

Nursu'aidah, H. 2014. Effects of fertilizer on root nodulation, chlorophyll content and nitrate reductase activity in long bean and mung bean. Tesis de maestría. Facultad de Ciencias, Universidad de Malaya, Kuala Lumpur. Malasia. Pp. 113.

de Paz, M. 2014. Heterogeneidad de micrositio, dinámica de nutrientes y facilitación en especies leñosas de los matorrales del NO de la Patagonia. Tesis Doctoral. Centro Regional Universitario Bariloche, Universidad Nacional del Comahue, San Carlos de Bariloche. Argentina. Pp. 281.

Prach, K., R. Marrs, P. Pyšsek, and R. van Diggelen. 2007. Manipulation of succession. Pp. 121-149 in L. R. Walker, J. Walker and R. J. Hobbs (eds.). Linking restoration and ecological succession. Springer, New York, New York, USA. https://doi.org/10.1007/978-0-387-35303-6 6.

Qasem, J. R. 2020. Weed Seed Dormancy: The Ecophysiology and Survival Strategies. Pp. 1-36 en J.C. Jimenez Lopez (ed.). Seed Dormancy and Germination. IntechOpen, London, UK.

Raffaele, E., T. T. Veblen, M. Blackhall, and N. Tercero-Bucardo. 2011. Synergistic influences of introduced herbivores and fire on vegetation change in northern Patagonia, Argentina. Journal of Vegetation Science 22:59-71. https://doi.org/ 10.1111/j.1654-1103.2010.01233.x.

Saruhan, V., A. Kusvuran, and K. Kokten. 2015. Effects of sewage sludge used as fertilizer on the yield and chemical contents of common vetch (Vicia sativa L.) and soil. Legume Research-An International Journal 38:488-495. https: //doi.org/10.5958/0976-0571.2015.00133.2.

Sawma, J. T., and C. L. Mohler. 2002. Evaluating seed viability by an unimbibed seed crush test in comparison with the Tetrazolium Test. Weed Technology 16:781-786. https://doi.org/10.1614/0890-037X(2002)016[0781: ESVBAU]2.0.CO;2.

Smýkal, P., V. Vernoud, M. W. Blair, A. Soukup, and R. D. Thompson. 2014. The role of the testa during development and in establishment of dormancy of the legume seed. Frontiers in Plant Science 5:1-19. https://doi.org/10.3389/ fpls.2014.00351.

Tang, L., C. Cheng, K. Wan, R. Li, D. Wang, Y. Tao, J. Pan, J. Xie, and F. Chen. 2014. Impact of fertilizing pattern on the biodiversity of a weed community and wheat growth. Plos One 9:e84370. https://doi.org/10.1371/ journal.pone.0084370.

Urretavizcaya, M. F., P. L. Peri, L. H. Monelos, H. Arriola, M. F. Oyharçabal, L. Contardi, M. Muñoz, E. Sepúlveda, and G. E. Defossé. 2018. Condiciones de suelo y vegetación en tres bosques quemados de Nothofagus pumilio en Argentina y experiencias para su restauración activa. Ecología Austral 28:383-399. https://doi.org/10.25260/EA.18.28.2.0.480.

Van Assche, J. A., K. L. A. Debucquoy, and W. A. F. Rommens. 2003. Seasonal cycles in the germination capacity of buried seeds of some Leguminosae (Fabaceae). New Phytologist 158:315-323. https://doi.org/10.1046/j.14698137.2003.00744.x.

Varela, S. A. 2005. Efecto del compost de biosólidos sobre la recuperación de un bosque quemado de Nothofagus pumilio (lenga). Tesis de licenciatura. Centro Regional Universitario Bariloche, Universidad Nacional del Comahue, Bariloche. Argentina. Pp. 79.

Varela, S. A., M. E. Gobbi, and F. Laos. 2006. Banco de semillas de un bosque quemado de Nothofagus pumilio: efecto de la aplicación de compost de biosólidos. Ecología Austral 16:63-78.

Varela, S. A., M. E. Gobbi, and F. Laos. 2011. Can biosolids compost improve, in the short term, native vegetation and soils fertility in burned Nothofagus pumilio forest in Patagonia, Argentina? Bosque 32:267-278. https://doi.org/10.4067/ S0717-92002011000300008.

Veblen, T. T., A. Holz, J. Paritsis, E. Raffaele, T. Kitzberger, and M. Blackhall. 2011. Adapting to global environmental change: what role for disturbance ecology? Austral Ecology 36:891-903. https://doi.org/10.1111/j.1442-9993.2010.02236.x.

Walter, I., and R. Calvo. 2009. Biomass production and development of native vegetation following biowaste amendment of a degraded, semi-arid soil. Arid land research and management 23:297-310. https://doi.org/10.1080/ 15324980903231827.

Wang, F., Z. Li, H. Xia, B. Zou, N. Li, J. Liu, and W. Zhu. 2010. Effects of nitrogen-fixing and non-nitrogen-fixing tree species on soil properties and nitrogen transformation during forest restoration in southern China. Soil Science and Plant Nutrition 56:297-306. https://doi.org/10.1111/j.1747-0765.2010.00454.x.

Wardle, D. A., and D. A. Peltzer. 2007. Aboveground-belowground linkages, ecosystem development, and ecosystem restoration. Pp. 45-68 in L. R. Walker, J. Walker and R. J. Hobbs (eds.). Linking restoration and ecological succession. Springer, New York, New York, USA. https://doi.org/10.1007/978-0-387-35303-6_3.

Wiggers, M. S., L. K. Kirkman, R. S. Boyd, and J. K. Hiers. 2013. Fine-scale variation in surface fire environment and legume germination in the longleaf pine ecosystem. Forest Ecology and Management 310:54-63. https://doi.org/ 10.1016/j.foreco.2013.07.030.

Zendejas, H. S. L., M. S. Oba, A. S. Oba, E. G. Gallegos, and J. L. G. Estrada. 2015. A bioeconomic approach for the production of biofertilizers and their influence on faba bean (Vicia faba L) productivity. Journal of Natural Sciences 3:75-91. https://doi.org/10.15640/jns.v3n2a5. 